\title{
Impacto da maturação sexual na força de membros superiores e inferiores em adolescentes
}

\author{
Impact of sexual maturation on upper and lower limb strength \\ on adolescents
}

1. Universidade Federal de Santa Catarina. Mestre em Educação Física. Florianópolis, SC. Brasil.

2. Universidade Federal de Sergipe. Departamento de Educação Física. Núcleo de Pesquisa em Aptidão Física de Sergipe. Sergipe, SE. Brasil

Recebido em 24/07/09 Revisado em 06/08/09 Aprovado em 17/09/09
Resumo - Estudos relacionados ao desempenho e à saúde, ao analisar parâmetros de força de membros superiores (FMMSS) e inferiores (FMMII), têm considerado a maturação biológica e sexual como uma das variáveis mais importantes na interpretação dos resultados, podendo o desempenho variar conforme o estágio maturacional. Sendo a menarca um marcador de maturação, o objetivo deste estudo foi comparar a FMMSS e FMMII em adolescentes que já apresentaram a menarca com aquelas que ainda não apresentaram. $\mathrm{O}$ estudo de delineamento transversal foi composto por 128 adolescentes de 11 a 14 anos, divididas em dois grupos: não maturadas $(n=72)$ e maturadas $(n=56)$. Para verificar a FMMSS, foi realizado o teste de preensão palmar por meio do dinamômetro manual. Já a FMMII foi verificada pelo teste de impulsão vertical. Para análise dos dados, foi usada a estatística descritiva e análise de covariância (ANCOVA), sendo as covariáveis desta análise a idade, peso corporal, estatura e IMC. Foi adotado um nível de significância de 5\% ( $<<0,05)$. A FMMSS diferiu entre as não maturadas $(17,8 \pm 4,4) \mathrm{kg} / \mathrm{f}$ e as maturadas $(21,5 \pm 3,9) \mathrm{kg} / \mathrm{f}(\mathrm{p}<0,05)$. Entretanto, ao anular o efeito das variáveis antropométricas, esta diferença desapareceu. Em contrapartida, não houve diferença na FMMII entre os grupos (não maturadas = 23,7 $\pm 6,7 \mathrm{~cm}$; maturadas $=24,1 \pm 6,7 \mathrm{~cm}$ ). A presença da menarca pode ser um dos eventos que provocou melhores desempenhos no teste de FMMSS, provavelmente, devido às variáveis antropométricas. No entanto, esta afirmação não pode ser inferida ao se analisar a FMMII.

Palavras-chave: Menarca; Adolescentes; Atividade motora; Força muscular.

Abstract - Biological and sexual maturation is one of the most important variables for the interpretation of the results of performance and health studies analyzing upper (ULS) and lower limb strength (LLS), with performance varying according to maturation stage. Since menarche is a marker of maturation, the aim of this study was to compare ULS and LLS between adolescents who already had their first menarche and those who did not. A cross-sectional study was conducted on 128 adolescents aged 11 to 14 years and divided into two groups: adolescents who did not report the presence of menarche $(n=72)$, adolescents who reported menarche $(n=56)$. ULS was evaluated by the hand grip test using a manual dynamometer. The vertical jump test was used for the evaluation of LLS. Descriptive statistics and covariance analysis (ANCOVA) were used for data analysis, with age, body weight, height and BMI as covariables. The level of significance was set at $5 \%(p<0.05)$. ULS differed between G1 $(17.8 \pm 4.4 \mathrm{~kg} / \mathrm{f})$ and G2 $(21.5 \pm 3.9$ $\mathrm{kg} / \mathrm{f})(\mathrm{p}<0.05)$. However, no difference in LLS was observed between groups (G1 = 23.7 \pm 6.7 $\mathrm{cm} ; \mathrm{G} 2=24.1 \pm 6.7 \mathrm{~cm}$ ). The presence of menarche might be one of the events improving the performance in the ULS test, but the same cannot be inferred when analyzing LLS.

Key words: Menarche; Adolescents; Motor activity; Muscle strength. 


\section{INTRODUÇÃO}

A adolescência pode ser entendida, simplesmente, como o período de transição entre a infância e a vida adulta. Entretanto, mais do que uma simples transição, é um período marcante do ciclo de vida, no qual ocorrem grandes transformações biológicas, psicológicas e sociais que afetam toda vida subsequente, tornando-se um dos períodos mais intensos e marcantes do ser humano.

Até a idade adulta, crianças e adolescentes passam por uma série de estágios que resultam em um grau crescente de maturação, caracterizando o processo de desenvolvimento do ser humano, o que, muitas vezes, não pode ser bem definido pela idade cronológica e, por isso, deve-se considerar aspectos biológicos e de avaliação temporal ${ }^{2-3}$. Dentre as diversas formas de avaliação deste processo, destaca-se a avaliação da maturação humana, como a idade gestacional, morfológica, dentária, óssea e o aparecimento de características sexuais secundárias ${ }^{1-4}$.

No sexo feminino, durante a adolescência, ocorre uma maior produção do hormônio estrogênio, responsável por estimular o crescimento dos órgãos genitais e o desenvolvimento da mama. Somando-se a isto, é nesta fase que ocorre o primeiro período menstrual (menarca) ${ }^{1}$. Este evento ocorre habitualmente após o pico de velocidade da estatura e quando a mama e a pilosidade púbica estão no nível 4 de maturação. Em relação à maturação óssea, a menarca ocorre, mais ou menos, no momento da fusão epifisária das falanges distais dos dedos que corresponde a uma idade óssea entre os 12 e os 14 anos e é condicionado por fatores genéticos, socioeconômicos, étnicos e morfológicos ${ }^{4}$.

Por estes fatores, o período de seu aparecimento varia, dependendo da localidade. Petroski et al. ${ }^{5}$ identificaram que, em média, a idade de menarca em escolares da grande Florianópolis (SC) é em torno de 12,6 anos. Leite et al. ${ }^{6}$ identificaram que em Campinas (SP) a média para a idade de menarca é de 12,3 anos. Cakir et al. ${ }^{7}$, na Turquia, identificaram que a primeira menstruação ocorre por volta dos 13 anos e na zona rural de Bangladesh, acontece aos 15 anos $^{8}$.

A menarca é utilizada como um marcador de maturação biológica das características sexuais secundárias, além de ser, após o nascimento, um dos mais marcantes fenômenos do desenvolvimento físico do ser humano ${ }^{1,6,9}$. Neste sentido, pode indicar a idade em que se processa a divisão celular, a quantidade de células que constituirão os diferentes tecidos, determinar a velocidade de mielinização dos neurônios corticais, coordenar o arranjo estru- tural do sistema nervoso e estabelecer o contexto para que a aprendizagem e o desenvolvimento da autoestima, do autoconceito e da responsabilidade se efetuem ${ }^{1-4,9,10}$. Assim, o estágio maturacional tem que ser levado em consideração ao se interpretar resultados de desempenho motor ou de aspectos da aptidão física e diversas manifestações do movimento corporal em geral ${ }^{9-12}$.

A força como um componente neuromuscular da aptidão física tem seu papel fundamental tanto para a promoção da saúde quanto para o sucesso em diversos esportes. Estando parado ou em movimento, este componente torna-se fundamental para as atividades físicas diárias em todas as fases da vida. Estudos relacionados ao desempenho e à saúde avaliaram a relação entre a idade de menarca e a performance motora, e concluíram que as jovens com atrasos maturacionais apresentavam melhores performances do que as com maturação normal em tarefas que implicassem velocidade de membros ${ }^{10}$. Em contrapartida, em uma pesquisa longitudinal, foi encontrado que melhores níveis de força foram evidenciados em adolescentes que já haviam tido a primeira menstruação $^{13}$. Outros pesquisadores que utilizaram diferentes marcadores de maturação sexual, como a pilosidade pubiana, encontraram que o desempenho, além de ser dependente do estágio maturacional para alguns testes motores, pode sofrer influências da faixa etária e variáveis antropométricas ${ }^{14}$.

Deste modo, controvérsias são encontradas na literatura acerca da inter-relação entre maturação e desempenho motor, o que demonstra a necessidade de pesquisas que controlem um maior número de variáveis possíveis. Além disso, uma pesquisa que traga informações a respeito desta relação possibilita a seleção dos estímulos adequados, de modo a não exigir comportamentos e desempenhos incompatíveis com o nível em que a criança e o adolescente se encontram, permite aos professores de Educação Física antecipar os fenômenos característicos das diferentes fases da adolescência, no que diz respeito à mobilidade dos indivíduos durante as aulas, facilitando a adequação do treinamento e da iniciação esportiva ao ritmo individual e segmentar do crescimento.

Assim, o presente estudo tem como objetivo comparar a força de membros superiores e inferiores das adolescentes que já apresentaram a menarca com aquelas que ainda não apresentaram.

\section{PROCEDIMENTOS METODOLÓGICOS}

Este estudo de delineamento transversal faz parte de um projeto de pesquisa intitulado "Caracte- 
rísticas do Crescimento, Composição Corporal e Desempenho Físico de Escolares Sergipanos - um estudo Longitudinal", coordenado pelo Núcleo de Pesquisa em Aptidão Física de Sergipe (NUPAFISE), do Departamento de Educação Física da Universidade Federal de Sergipe (UFS), e aprovado pelo Comitê de Ética desta instituição (CAAE 0035.0.0107.000-06).

Dentre os objetivos deste projeto, está a realização de avaliações físicas anuais em todos os alunos ingressantes no ensino fundamental do Colégio de Aplicação da UFS, desde o ano de 2004. Aproximadamente, 360 alunos já foram avaliados desde o início do projeto, sendo que, em torno de 50\%, são estudantes do sexo feminino. Deste percentual, 130 garotas foram avaliadas em 2005 e 2006 e, devido aos testes físicos que foram realizados naqueles anos, optou-se por este grupo de adolescentes para o presente estudo.

Das 130 garotas que foram selecionadas para a avaliação, duas não levaram o Termo de Consentimento Livre e Esclarecido (TCLE) assinado pelos pais/responsáveis, ficando excluídas do estudo. Assim, a amostra da presente investigação foi formada por 128 garotas de 11 a 14 anos de idade. A amostra foi dividida em dois grupos: não maturadas, formado pelas adolescentes que não reportaram a presença da menarca ( $n=72)$; e as maturadas, composto por aquelas que reportaram a ocorrência da menarca $(n=56)$.

Foi realizado o cálculo do tamanho da amostra a posteriori aplicação dos testes de força no programa Stata 9.0, levando-se em consideração os seguintes parâmetros: nível de significância de 95\%, um poder do teste para comparação de duas médias de 0,90, uma razão de 2:1. Após os cálculos, foi evidenciado que seriam necessários 42 adolescentes no grupo das não maturadas e 21 no das maturadas. Desta forma, em ambos os grupos, foram avaliadas mais adolescentes do que o estimado pelo cálculo amostral para extrapolação dos dados.

Para saber se as adolescentes já haviam tido a menarca, utilizou-se o método retrospectivo. Um dos avaliadores da equipe, que foi composta por cinco professores e seis acadêmicos de Educação Física, ficou responsável por perguntar às garotas se elas já haviam apresentado a primeira menstruação. Este avaliador foi selecionado por ser o mais treinado e experiente em termos acadêmicos relacionados com a temática, pois ao se tratar esse assunto com as jovens, é necessário um procedimento de abordagem adequado.

Os dados foram coletados no laboratório de aptidão física do NUPAFISE na UFS. Inicialmente, coletou-se o peso corporal, por meio de uma balança digital, da marca Techline ${ }^{\circledR}$, com precisão de 100 gramas e a estatura, usando um estadiômetro profissional portátil da marca Sanny ${ }^{\circledR}$, com precisão de 0,1 centímetros. Com estes dados, calculou-se o índice de massa corporal (IMC). Todas as medidas seguiram as padronizações de Alvarez e Pavan ${ }^{15}$.

Para a realização dos testes físicos, primeiramente, entrou no laboratório o Grupo de não maturadas, depois o Grupo de maturadas. Sendo que cada integrante dos grupos foi avaliada de maneira individual para evitar um ambiente competitivo no local.

Para verificar a força de membros superiores, utilizou-se o teste de preensão palmar, que tem muitas aplicações clínicas diferentes, sendo usado, por exemplo, como um indicador de força total de membros superiores e, em diversos testes, de aptidão física $^{16-17}$. Como instrumento de avaliação desta capacidade física, foi usado o dinamômetro manual Jamar ${ }^{\circledR}$, que apresenta uma escala de força descrita até 200 libras e/ou 90 kg/f - Quilograma/força. Para a coleta da força, foi usada a padronização recomendada por Matsudo ${ }^{18}$ empregada para adolescentes. $\mathrm{O}$ teste foi executado com o avaliado na posição em pé e o braço estendido ao lado do corpo. A medição da força foi realizada com três repetições, alternando o membro avaliado, sendo, inicialmente, testada a mão direita e, logo em seguida, a mão esquerda. $\mathrm{O}$ intervalo de tempo entre uma tentativa e outra foi de um minuto, havendo, desta forma, o controle da fadiga muscular ${ }^{19}$. Foi solicitado que em cada medição fosse realizada uma preensão máxima, com duração de cinco segundos. Foi considerado o valor mais elevado das três tentativas para o lado direito e para o lado esquerdo e considerado como valor final a sua média.

Para a verificação da força de membros inferiores, foi realizado o teste de impulsão vertical, conforme as padronizações de Matsudo ${ }^{18}$. Este teste avalia a força explosiva destes membros ${ }^{20}$. Para este teste, é fixada uma fita métrica em toda a altura de uma parede lisa, do solo ao teto, para marcar a distância do salto do avaliado. $\mathrm{O}$ avaliado se posiciona com o lado direito do corpo junto à parede, alinhado à fita métrica, de modo que o braço direito fique estendido para cima, no prolongamento do corpo, tentando alcançar uma altura máxima com a ponta dos dedos, estando os pés plantados no solo. Nesta altura máxima é demarcada uma primeira marca. Ao sinal do avaliador, é realizada uma impulsão vertical máxima, tendo o avaliado que alcançar, com os dedos da mão, uma altura máxima. Após a realização do salto, é demarcada 
uma segunda marca e a distância em centímetros entre a primeira marca e a segunda, equivalente à distância alcançada. Foram realizados dois saltos, com intervalo de um minuto entre os mesmos, sendo computado o valor do melhor salto.

Antes de iniciar os testes, todos os participantes foram orientados, detalhadamente, quanto à execução dos mesmos, realizando uma medida de preensão palmar para cada membro e um teste de impulsão vertical para a familiarização com a técnica, evitando viés nas mensurações, devido às influências no aspecto ensino-aprendizagem.

Inicialmente, a normalidade dos dados foi testada pelo teste de Kolmogorov-Smirnov. Foi utilizada a estatística descritiva, descrevendo a idade e as variáveis antropométricas em valores de média, desvio padrão (DP) e erro padrão (EP). Foi verificada a interação entre a presença/ausência da menarca com a força de membros superiores e inferiores. Como não houve interação entre as variáveis, usou-se a análise de covariância (ANCOVA) para comparar a força das adolescentes de ambos os grupos, tendo como as covariáveis (variáveis controladas) a idade (Modelo 1), o peso corporal, a estatura e o IMC (Modelo 2), evitando qualquer influência nos testes de força. As análises respeitaram um nível de significância de 5\% (p<0,05).

\section{RESULTADOS}

A tabela 1 descreve os valores médios da idade, do peso, da estatura, do IMC e da força dos membros superiores e inferiores. Observa-se que as maturadas apresentaram maiores valores para a idade, variáveis antropométricas e força de membros superiores $(\mathrm{p}<0,05)$.

$\mathrm{Na}$ tabela 2, verificam-se os valores da média e do erro padrão, após a ANCOVA para os testes físicos de força para membros superiores e inferiores. No modelo 1 , que teve somente a idade sendo controlada, foi verificado que os grupos diferiram significativamente para força de membros superiores. Ao controlar a idade e as variáveis antropométricas (Modelo 2), não foi evidenciado nenhuma diferença para a força de membros superiores e inferiores entre os grupos $(\mathrm{p}>0,05)$.

\section{DISCUSSÃO}

O presente estudo de delineamento transversal objetivou comparar a força de membros superiores e inferiores em adolescentes que já apresentaram a menarca, com aquelas que ainda não apresentaram, contribuindo, assim, para a área de crescimento físico, desenvolvimento e maturação humana, no que diz respeito às variáveis de desempenho

Tabela 1. Valores médios e o desvio padrão para a idade, variáveis antropométricas e para força.

\begin{tabular}{|c|c|c|c|c|}
\hline \multirow[t]{2}{*}{ Variáveis } & \multirow{2}{*}{$\begin{array}{c}\text { Toda a amostra } \\
\qquad \begin{array}{c}(\mathrm{n}=128) \\
\bar{X}(\mathrm{DP})\end{array}\end{array}$} & \multirow{2}{*}{$\begin{array}{l}\text { Não maturadas } \\
\qquad \begin{array}{c}(\mathrm{n}=72) \\
\bar{X}(\mathrm{DP})\end{array}\end{array}$} & \multirow{2}{*}{$\begin{array}{l}\text { Maturadas } \\
(\mathrm{n}=56) \\
\bar{X}(\mathrm{DP})\end{array}$} & \multirow{2}{*}{ p-valor } \\
\hline & & & & \\
\hline Idade (anos) & $12,1(0,9)$ & $11,7(0,8)$ & $12,6(0,9)$ & $<0,001^{*}$ \\
\hline Peso (kg) & $44,9(12)$ & $39,8(10,5)$ & $51,4(10,6)$ & $<0,001^{*}$ \\
\hline Estatura $(\mathrm{cm})$ & $151,4(8,5)$ & $147,4(8,5)$ & $156,7(5,2)$ & $<0,001^{*}$ \\
\hline FMMSS $(\mathrm{kg} / \mathrm{f})$ & $19,4(4,6)$ & $17,8(4,4)$ & $21,5(3,9)$ & $<0,001^{*}$ \\
\hline FMMII (cm) & $23,9(6,7)$ & $23,7(6,7)$ & $24,1(6,7)$ & 0,716 \\
\hline
\end{tabular}

$\bar{X}$ - média; DP - desvio padrão; FMMSS - força de membros superiores; FMMII - força de membros inferiores; *p<0,05.

Tabela 2. Valores de média, erro padrão e o resultado da análise de covariância (F) entre os grupos, tendo a idade (Modelo 1), idade, peso, estatura e o IMC (Modelo 2) como co-variáveis.

\begin{tabular}{|c|c|c|c|c|c|c|c|c|c|c|}
\hline \multirow{3}{*}{ Força } & \multicolumn{4}{|c|}{ Modelo 1} & \multirow{3}{*}{$\mathrm{F}$} & \multicolumn{4}{|c|}{ Modelo 2} & \multirow{3}{*}{$\mathrm{F}$} \\
\hline & \multicolumn{2}{|c|}{ Não maturadas } & \multicolumn{2}{|c|}{ Maturadas } & & \multicolumn{2}{|c|}{ Não maturadas } & \multicolumn{2}{|c|}{ Maturadas } & \\
\hline & $\bar{X}$ & $\mathrm{EP}$ & $\bar{X}$ & $\mathrm{EP}$ & & $\bar{X}$ & $\mathrm{EP}$ & $\bar{X}$ & EP & \\
\hline MMSS (kg/f) & 18,0 & 0,5 & 21,2 & 0,6 & $14,5^{*}$ & 19,3 & 0,4 & 19,6 & 0,5 & 0,2 \\
\hline MMII (cm) & 24,2 & 0,8 & 23,5 & 1,0 & 0,3 & 24,3 & 0,9 & 23,3 & 1,0 & 0,5 \\
\hline
\end{tabular}

Modelo 1 - ANCOVA tendo como covariável a Idade.

Modelo 2 - ANCOVA tendo como covariável a idade, peso, estatura, índice de massa corporal. MMSS - Membros Superiores; MMII - Membros Inferiores; $\bar{X}$ - média; EP - erro padrão; F - Valor do teste da ANCOVA; *p $<0,05$. 
físico de jovens. Além disso, traz informações relevantes para professores e profissionais da área de Educação Física que trabalham com jovens nas diversas manifestações do movimento corporal e desempenho motor.

A força dos membros superiores reflete uma dimensão funcional importante no crescimento, pois é uma variável que sofre dramáticas mudanças com o avanço da idade, principalmente, na segunda década da vida ${ }^{21}$. Embora seja comum a avaliação da força de preensão palmar em esportes que a exigem com mais especificidade, esta é uma característica de força que vem começando a ser mais pesquisada, inclusive, comparando-a com a aptidão física relacionada à saúde, devido à evidente associação da força de preensão manual com diversas atividades do cotidiano ${ }^{22}$.

No presente estudo, foi encontrado que as estudantes que apresentaram a menarca tiveram melhor desempenho no teste de dinamometria manual do que aquelas que ainda não haviam apresentado ( $\mathrm{Ta}-$ bela 1). Resultados semelhantes foram reportados em outras pesquisas no Brasil e na África ${ }^{13,24,25}$.

Matsudo et al. ${ }^{24}$ encontraram que meninas do estado de São Paulo, que já tinham apresentado menarca, apresentavam força de preensão palmar, mensurada pelo dinamômetro manual, superiores às meninas que não haviam apresentado menarca, de mesma idade cronológica. $\mathrm{O}$ mesmo fenômeno foi observado em Moçambique, na África, por meio da pesquisa realizada por Prista et al..$^{25}$, com meninas de 8 a 17 anos.

Biassio et al..$^{13}$, em pesquisa longitudinal com escolares de 8 a 18 anos, de Ilha Bela (SP), observaram um aumento gradual e significativo da força de membros superiores, em função do período maturacional, sendo que, no período de dois anos antes da menarca, até dois anos após a menarca, foram evidenciados os maiores aumentos para esta variável analisada pela força de preensão palmar (73,1\%).

Ao verificar se algumas variáveis interferiam na força de membros superiores (Tabela 2), o presente estudo encontrou que, ao controlar (anular) o efeito da idade (Modelo 1), a diferença entre as jovens maturadas e não maturadas se manteve, reforçando a idéia de que independente da faixa etária, ocorrem mudanças significativas na força de preensão manual entre adolescentes maturadas e não maturadas, tendo as primeiras melhor desempenho que as segundas. Ao anular o efeito das variáveis antropométricas (Modelo 2), as diferenças que existiam para a força de membros superiores não permaneceram. Estes dados sugerem que variá- veis como peso corporal, estatura e IMC interferem no desempenho deste teste motor. Uma possível justificativa para estes achados é que tais variáveis estão associadas com a presença da menarca, pois adolescentes com déficit em peso corporal e estatura apresentam um retardo da menarca e um desempenho mais baixo em testes de força ${ }^{1,4,14}$.

Neste sentido, a maturação sexual é uma variável que pode influenciar a força de membros superiores em adolescentes, e este fator tem que ser levado em consideração nos diversos aspectos que envolvem a vida destes jovens. Como exemplo, pode-se citar as diversas modalidades esportivas cujos membros superiores têm atuação principal, fazendo-se necessário uma divisão em categorias não somente por idade cronológica, mas também e sobretudo, por nível maturacional.

Em relação à força de membros inferiores, não houve diferenças significativas no desempenho deste teste entre os dois grupos (Tabela 1 e 2). Resultado semelhante foi reportado em outros estudos $^{14,23,26}$. Andrade ${ }^{23}$, ao comparar a força de membros inferiores de adolescentes do sexo feminino de diferentes estágios maturacionais (pós-púbere, púbere e pré-púbere), não encontrou diferenças no desempenho deste teste. Quatman et al. ${ }^{26}$ verificaram a força de membros inferiores, durante dois anos, em adolescentes atletas que, no primeiro ano, estavam classificados no estágio maturacional púbere e, no segundo ano de estudo, no pós-púbere. Os autores encontraram que os atletas do sexo masculino demonstraram um aumento significativo na altura do salto vertical, contudo, isto não foi observado no sexo feminino. Ulbrich et al..$^{14}$, ao investigar a força explosiva de jovens eutróficos com a mesma faixa etária do presente estudo, também não encontraram diferenças entre os adolescentes de diferentes estágios maturacionais.

Outras investigações encontraram resultados contrários ao do presente estudo em relação à força de membros inferiores ${ }^{13,24-25}$. Biassio et al. ${ }^{13}$, em uma pesquisa longitudinal, encontraram diferenças na força de membros inferiores, avaliada pelo teste de impulsão vertical, entre o período pré-menarca e o pós-menarca, entre escolares de Ilha Bela (SP). No entanto, ressaltou que esta diferença foi menor do que comparada às alterações que ocorreram na força de membros superiores entre os mesmos grupos.

Assim, não há um consenso na literatura acerca de quem pode apresentar um melhor desempenho para força de membros inferiores no sexo feminino, quando os estágios de maturação são próximos, como na menarca, que não leva em consideração 
outros fatores maturacionais que podem influenciar os resultados, como o crescimento somático. $\bigcirc$ que se sabe é que, durante a puberdade, não há incrementos significativos na força de salto vertical em adolescentes do sexo feminino que não praticam alguma atividade física específica para este fim, vindo apresentar um pico de força de membros inferiores somente por volta dos 16 anos de idade $\mathrm{i}^{10,27-28}$.

Os achados do presente estudo podem contribuir para profissionais e professores de educação física que trabalham com jovens adolescentes em aulas de Educação Física, iniciação esportiva e treinamento esportivo, pois traz informações do impacto da menarca na força, evidenciando que este indicador de maturação sexual tem que ser levado em consideração para o desempenho em testes motores, facilitando a adequação do treinamento e da iniciação esportiva ao ritmo individual de cada adolescente.

Este estudo apresenta algumas limitações que necessitam ser citadas, como o fato de ter sido realizado com estudantes de uma única instituição de ensino, não podendo ser generalizado para estudantes de outras escolas que podem praticar atividades físicas específicas para o desenvolvimento da força dos membros avaliados. Entretanto, em função da variável força ser muito relacionada com o processo de crescimento e desenvolvimento humano, os resultados do atual estudo podem retratar o que acontece com adolescentes em geral. Cita-se, também, como limitação a não identificação de quanto tempo a menarca havia ocorrido nas adolescentes maturadas, pois pode-se colocar em um mesmo grupo jovens que já apresentaram a menarca, há muito tempo, com aquelas que recentemente apresentaram, podendo outros fatores maturacionais virem a influenciar a variável força.

Pontos fortes do presente estudo necessitam ser destacados como, por exemplo, o tratamento estatístico que controlou possíveis variáveis que poderiam interferir nos resultados dos testes motores, como a idade, o peso, a estatura e o IMC. Este controle foi realizado pela ANCOVA, um método aplicado para controlar as variáveis intervenientes da variável dependente (força). A partir desta análise, qualquer diferença encontrada é dada, exclusivamente, pela relação entre as variáveis dependente e independente (maturação). Outros autores utilizaram este método para fazer esta relação ${ }^{12}$.

\section{CONCLUSÃO}

Com os dados do presente estudo, pode-se concluir que, para a força de membros superiores, as ado- lescentes maturadas apresentaram maiores níveis de força do que seus pares que ainda não maturaram, sendo este achado influenciado por variáveis antropométricas. Em relação à força de membros inferiores, não houve diferenças quanto aos níveis de maturação sexual.

Desta forma, recomenda-se que futuros estudos investiguem a influência da menarca em mais aspectos da aptidão física, de maneira longitudinal, para identificar as possíveis relações de causalidade entre as variáveis.

\section{REFERÊNCIAS BIBLIOGRÁFICAS}

1. Malina RM, Bouchard C, Bar-Or O. Growth, Maturation, and Physical Activity. Champaign, IL: Human Kinetics, 2004.

2. Cacciari E, Milani S, Balsamo A, Dammacco F, De Luca F, Chiarelli F, Pasquino AM, Tonini G, Vanelli M. Italian cross-sectional growth charts for height, weight and BMI (6-20 y). Eur J Clin Nutr 2002;56(2):171-180.

3. Rogol AD, Clark PA, Roemmich JN. Growth and pubertal development in children and adolescents: effects of diet and physical activity. Am J Clin Nutr 2000;72 (Suppl 2):521S-528S.

4. Vieira F, Fragoso I. Morfologia e Crescimento. $2^{\text {a }}$ Edição. Cruz Quebrada: Faculdade de Motricidade Humana - Universidade Técnica de Lisboa, 2006.

5. Petroski EL, Velho NM, De Bem MFL. Idade de menarca e satisfação com o peso corporal. Rev Bras Cineantropom Desempenho Hum 1999;1(1):30-36.

6. Leite RMC, Buoncompagno EM, Leite ACC, Mergulhão EA, Battistoni MM. Psychosexual characteristics of female university students in Brazil. Adolescence 1994;29(114):399-460.

7. Cakir M, Mungan I, Karakas T, Girisken I, Okten A. Menstrual pattern and common menstrual disorders among university students in Turkey. Pediatr Int 2007;49(6):938-942.

8. Bosch AM, Willekens FJ, Baqui AH, Van Ginneken JK, Hutter I. Association between age at menarche and early-life nutritional status in rural Bangladesh. J Biosoc Sci 2008; 40(2):223-237.

9. Semiz S, Kurt F, Kurt DT, Zencir M, SevinçO. Pubertal development of Turkish children. J Pediatr Endocrinol Metab 2008;21(10):951-961.

10. Beunen G, Malina RM. Growth and physical performance relative to the timing of the adolescent spurt. Exerc Sport Sci Rev 1988;16(1):503-540.

11. Naughton G, Farpour-Lambert NJ, Carlson J, Bradney M, Van Praagh E. Physiological issues surrounding the performance of adolescent athletes. Sports Med 2000;30(5):309-325.

12. Jones MA, Hitchen PJ, Stratton G. The importance of considering biological maturity when assessing physical fitness measures in girls and boys aged 10 to 16 years. Ann Hum Biol 2000;27(1):57-65. 
13. Biassio LG, Matsudo SMM, Matsudo VKR. Impacto da menarca nas variáveis antropométricas e neuromotoras da aptidão física, analisado longitudinalmente. Rev Bras Ciên Mov 2004;12(2):97-101.

14. Ulbrich AZ, Bozza R, Machado HS, Michelin A, Vasconcelos IQA, Stabelini Neto A, Mascarenhas LPG, Campos W. Aptidão física em crianças e adolescentes de diferentes estágios maturacionais. Fit Perf J 2007;6(5): 277-282.

15. Alavarez BR, Pavan AL. Alturas e comprimentos. In: Petroski EL (Ed). Antropometria: Técnicas e padronizações. Blumenal: Nova Letra, 2007. $3^{\mathrm{a}}$ ed. Cap.2, p. 31-44.

16. Balogum JA, Akomolafe CT, Amusa LO. Grip strength: effects of testing posture and elbow position. Arch Phys Med Rehabil 1991;72(5):280-283.

17. Moreira D, Alvarez RR, Godoy JR, Cambraia AN. Abordagem sobre preensão palmar utilizando o dinamômetro Jamar®: uma revisão de literatura. Rev Bras Ciên Mov 2003;11(2):95-99.

18. Matsudo VKR. Testes em Ciências do Esporte. 7 ed. Londrina: Midiograf 2005.

19. Firrel JC, Crain GM . Which setting of the dynamometer provide s maximal grip strength? J Hand Surg 1996; 21A(3):397-401.

20. Battista RA, Pivarnik JM, Dummer GM, Sauer N, Malina RM. Comparisons of physical characteristics and performances among female collegiate rowers. J Sports Sci 2007;25(6):651-657.

21. Giarolla RA, Figueira Junior A, Matsudo VKR. Análise da força da mão dominante em relação à mão nãodominante em escolares de 8 a 18 anos. Rev Bras Ciên Mov 1991;5(1):31-39.

22. Bertuzzi RCM, Franchini E, Kiss MAPD. Análise da força e da resistência de preensão manual e as suas relações com variáveis antropométricas em escaladores esportivos. Rev Bras Ciên Mov 2005;13(1):87-93.

23. Andrade EL. Atividade física, aptidão física, consumo alimentar e maturação sexual em adolescentes de uma escola pública de Ilhabela - SP. [Dissertação Mestrado - Programa de Pós Graduação em Saúde Pública]. São Paulo (SP): Universidade de São Paulo; 2001.

24. Matsudo VKR, França NM, Matsudo SMM. Quantitative and qualitative differences in motor performance in adolescent brazilian girls. In: França N. Criança e exercício I: aptidão física e maturação sexual. São Caetano do Sul: CELAFISCS, 1994. p. 154-156.

25. Prista A, Maia J, Saranga S, Marques A. Saúde, Crescimento e Desenvolvimento. Um estudo epidemiológico em crianças e jovens de Moçambique. Lisboa: Fundação Galouste Gulbenkian, 2002.

26. Quatman CE, Ford KR, Myer GD, Hewett TE. Maturation leads to gender differences in landing force and vertical jump performance: a longitudinal study. Am J Sports Med 2006;34(5):806-813.

27. Martin RJ, Dore E, Twisk J, Van Praagh E, Hautier CA, Bedu M. Longitudinal changes of maximal short-term peak power in girls and boys during growth. Med Sci Sports Exerc 2004; 36(3):498-503.

28. Kellis E, Tsitskaris GK, Nikopoulou MD, Mousikou KC. The evaluation of jumping ability of male and female basketball players according to their chronological age and major leagues. J Strength Cond Res 1999;13 (1):40-46.

\section{Endereço para correspondência}

Antonio Cesar Cabral de Oliveira

Universidade Federal de Sergipe, Sergipe, SE. Brasil.

Centro de Ciências Biológicas e da Saúde.

Departamento de Educação Física

Campus Universitário. Rosa Elze. São Cristovão

49100-000 - Sergipe, SE. Brasil

E-mail: cabral@infonet.com.br 